\title{
Observation of second- and higher-order electric quadrupole interactions with an atomic ion
}

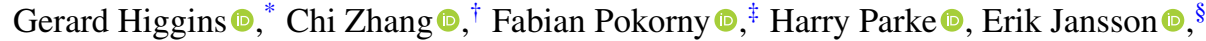 \\ Shalina Salim $\odot$, and Markus Hennrich @ 11 \\ Department of Physics, Stockholm University, SE-106 91 Stockholm, Sweden
}

(Received 6 May 2020; accepted 7 July 2021; published 2 August 2021)

\begin{abstract}
We observe an atomic ion's response to an electric quadrupole field to second and higher orders; this arises from the ion's electric quadrupole polarizability and hyperpolarizabilities. We probe a single ${ }^{88} \mathrm{Sr}^{+}$ion which is confined in the electric fields of a Paul trap and excited to Rydberg states. The quadrupolar trapping fields cause atomic energy level shifts and give rise to spectral sidebands. The effects on Rydberg $S_{1 / 2}$ states are described well by second-order perturbation calculations. The stronger effects on Rydberg $P_{1 / 2}$ states are captured by full diagonalization of the Hamiltonian.
\end{abstract}

DOI: 10.1103/PhysRevResearch.3.L032032

\section{INTRODUCTION}

Second- and higher-order quadrupole responses contribute to interatomic [1,2] and intermolecular [3] interactions; they affect crystal structures [4], surface and bulk phonons [5], the chemistry of solvents [6], and the behavior of nanoantennas [7]. Quadrupole effects are well known in trapped ion systems, where strong electric quadrupole fields provide confinement. First-order quadrupole shifts (due to the relatively weak static quadrupole field) affect trapped ion atomic clocks and precision spectroscopy experiments [8]. Second-order shifts (due to all of the trapping quadrupole fields) may become relevant in improved trapped ion precision experiments [9], and may affect transitions involving $F>\frac{1}{2}$ states at the $10^{-18}$ level [10], as shown in the Supplemental Material [11]. In addition, the Sr optical lattice clock is predicted to be sensitive to secondorder ac quadrupole shifts due to the lattice laser fields at the $10^{-19}$ level [12].

Rydberg states of atoms, ions, and molecules are highly excited states which are extremely sensitive to external fields. Various experiments are emerging which involve Rydberg states in strong quadrupole trapping fields: hybrid systems

\footnotetext{
*Present address: Institute for Quantum Optics and Quantum Information, Austrian Academy of Sciences, Vienna, Austria; higgins@chalmers.se

Present address: Centre for Cold Matter, Imperial College London, London, UK; c.zhang@imperial.ac.uk

*Present address: Department of Physics, University of Oxford, Clarendon Laboratory, Parks Road, Oxford OX1 3PU, England, United Kingdom.

${ }^{\S}$ Present address: Physikalisch-Technische Bundesanstalt, DE38116 Braunschweig, Germany.

"markus.hennrich@fysik.su.se
}

Published by the American Physical Society under the terms of the Creative Commons Attribution 4.0 International license. Further distribution of this work must maintain attribution to the author(s) and the published article's title, journal citation, and DOI. of neutral Rydberg atoms and trapped ions allow atom-ion interactions to be studied $[13,14]$, Rydberg molecules can be sensitively detected using an ion trap [15], trapped Rydberg ions have recently shown potential for quantum computing [16], and precision spectroscopy of Rydberg systems could be used to search for a fifth fundamental force [17]. The ion trap's electric fields cause extreme Stark effects in these systems, which have been extensively studied previously $[13,14,18$ 22]. In trapped Rydberg ion systems Stark effects can be diminished by minimizing the dipole electric field experienced by the ions (by cooling the ion motion to the ground state $[18,20]$ and by minimizing ion micromotion [21]), or else by reducing the Rydberg dipole polarizability using microwave dressing [23,24].

In this Rapid Communication we study quadrupole effects on Rydberg ions. Rydberg states in ion traps can display giant first-order quadrupole effects $[18,20]$. These effects are diminished by using $J=\frac{1}{2}$ Rydberg states, which do not have permanent quadrupole moments. However, quadrupole effects cannot be removed entirely; here we investigate second- and higher-order quadrupole effects displayed by a trapped ion in $J=\frac{1}{2}$ Rydberg states due to the states' quadrupole polarizabilities and hyperpolarizabilities. We observe that the quadrupole trapping fields cause energy level shifts, and that the oscillating energy level shift due to the oscillating trapping field gives rise to spectral sidebands. These effects will need to be considered in future experiments involving neutral Rydberg atoms, molecules, or ions in electric quadrupole traps.

\section{THEORETICAL BACKGROUND}

An external electric potential $\Phi$ perturbs a distribution of charges by

$$
H^{\prime}=\sum_{i} q_{i} \Phi=\sum_{i l m} q_{i} \phi_{l}^{m} r^{l} Y_{l}^{m}(\theta, \phi)
$$

where $q_{i}$ are the charges, $\Phi$ is expanded in terms of spherical harmonics $Y_{l}^{m}(\theta, \phi), \phi_{l}^{m}$ are scalars, and $\{r, \theta, \phi\}$ are the spherical coordinates. The energy shift caused by $H^{\prime}$ on a 
charge distribution may be described perturbatively [25]:

$$
E^{\prime}=-\sum_{l m} Q_{l}^{m} \phi_{l}^{m}-\sum_{l_{1} l_{2} m_{1} m_{2}} \alpha_{l_{1} l_{2}}^{m_{1} m_{2}} \phi_{l_{1}}^{m_{1}} \phi_{l_{2}}^{m_{2}}+\mathcal{O}\left(\phi^{3}\right)
$$

where $Q_{l}^{m}$ is the $l$ th multipole moment, $\alpha_{l_{1} l_{2}}^{m_{1} m_{2}}$ is the multipole polarizability, and the $\mathcal{O}$ represents higher-order terms. Formulas describing $Q_{l}^{m}$ and $\alpha_{l_{1} l_{2}}^{m_{1} m_{2}}$ are given in the Supplemental Material [11].

$Q_{1}^{m}$ describes the dipole moment, which gives rise to the linear Stark effect, and $\alpha_{11}^{m_{1} m_{2}}$ describes the dipole polarizability, which gives rise to the second-order (quadratic) Stark effect. $Q_{2}^{m}$ describes the quadrupole moment, which gives rise to the first-order quadrupole shift, which is well known in trapped ion precision spectroscopy when states with angular momentum $J>\frac{1}{2}$ are involved [8]. In this Rapid Communication we present second-order quadrupole effects on an atomic ion, which arise from the action of a quadrupole field on the ion's quadrupole polarizability $\alpha_{22}^{m_{1} m_{2}}$.

In our experiment the perturbing electric potential is the trapping potential of a linear Paul trap. The approximate electric potential close to the trap center is

$$
\begin{aligned}
\Phi= & \left(\phi_{\mathrm{rf}} \cos \Omega t+\phi_{\mathrm{rad}}\right) r^{2}\left[Y_{2}^{2}(\theta, \phi)+Y_{2}^{-2}(\theta, \phi)\right] \\
& +\phi_{\mathrm{ax}} r^{2} Y_{2}^{0}(\theta, \phi)
\end{aligned}
$$

where $\Omega$ is the frequency of the oscillating quadrupole field, $\phi_{\mathrm{rf}}$ describes its strength, the $\phi_{\mathrm{ax}}$ term provides axial confinement, the $\phi_{\text {rad }}$ term causes the radial nondegeneracy, $\theta$ is relative to the trap symmetry axis, and we consider the case where the trap symmetry axis is collinear with the magnetic field axis. When the trap symmetry axis is not collinear with the magnetic field axis, rotation between the coordinate systems defined by these axes is achieved using the Wigner D-matrix, as in [8]. $\left\{\phi_{\mathrm{rf}}, \phi_{\mathrm{ax}}, \phi_{\mathrm{rad}}\right\}$ are related to the ion's secular frequencies $\left\{\omega_{x}, \omega_{y}, \omega_{z}\right\}$ by

$$
\begin{aligned}
\phi_{\mathrm{rf}} & =\sqrt{\frac{2 \pi}{15}} \frac{1}{q} M \Omega \sqrt{\omega_{x}^{2}+\omega_{y}^{2}+\omega_{z}^{2}}, \\
\phi_{\mathrm{ax}} & =\sqrt{\frac{\pi}{5}} \frac{1}{q} M \omega_{z}^{2}, \\
\phi_{\mathrm{rad}} & =\sqrt{\frac{\pi}{30}} \frac{1}{q} M\left(\omega_{x}^{2}-\omega_{y}^{2}\right)
\end{aligned}
$$

where $M$ is the ion mass and $q$ is the elementary charge.

In this potential a $J \leqslant \frac{1}{2}$ state, which has a quadrupole polarizability $\quad \alpha_{22} \equiv \alpha_{22}^{00}=-\frac{1}{2}\left(\alpha_{22}^{1-1}+\alpha_{00}^{-11}\right)=\frac{1}{2}\left(\alpha_{22}^{2-2}+\right.$ $\left.\alpha_{22}^{-22}\right)$ and no quadrupole moment $\left(Q_{2}^{m}=0\right)$, is shifted by [26]

$$
\begin{gathered}
E^{\prime}=\alpha_{22}\left(\phi_{\mathrm{rf}}^{2} \cos 2 \Omega t+4 \phi_{\mathrm{rf}} \phi_{\mathrm{rad}} \cos \Omega t\right. \\
\left.+\phi_{\mathrm{rf}}^{2}+2 \phi_{\mathrm{rad}}^{2}+\phi_{\mathrm{ax}}^{2}\right)+\mathcal{O}\left(\phi^{3}\right) \\
\approx E_{2}^{\prime} \cos 2 \Omega t+E_{1}^{\prime} \cos \Omega t+E_{0}^{\prime}
\end{gathered}
$$

where the $\mathcal{O}$ represents the higher-order response to quadrupole fields, and we introduce $E_{0}^{\prime}, E_{1}^{\prime}$, and $E_{2}^{\prime}$ for clarity.

The static energy shift $E_{0}^{\prime}$ causes resonance frequency shifts, which depend on the field strengths, while the oscillating energy shifts $E_{1}^{\prime}$ and $E_{2}^{\prime}$ give rise to spectral sidebands.

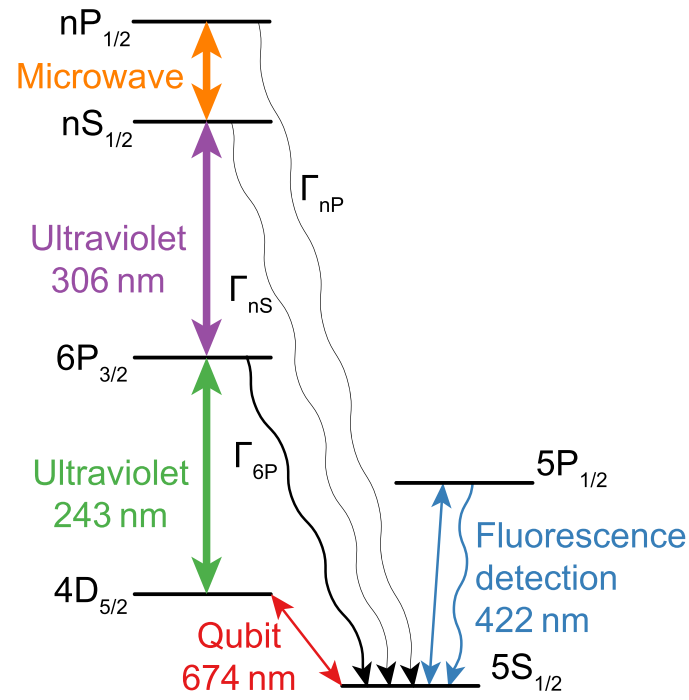

FIG. 1. Relevant level scheme of ${ }^{88} \mathrm{Sr}^{+}$. We probe the excitation spectra of Rydberg $S_{1 / 2}$ and $P_{1 / 2}$ states. We prepare ions in a Zeeman sublevel of $4 D_{5 / 2}$ using the "qubit" laser field, and we probe Rydberg $S_{1 / 2}$ and $P_{1 / 2}$ states using two UV laser fields, as well as a microwave field. Spectroscopy signals rely on decay from $6 P_{3 / 2}, n S_{1 / 2}$, and $n P_{1 / 2}$ to the ground state $5 S_{1 / 2}$. Scattering of 422-nm fluorescence light is used to distinguish whether the ion is in the initial state $4 D_{5 / 2}$ or the ground state $5 S_{1 / 2}$.

\section{METHODS}

We observe both the resonance shifts [due to $E_{0}^{\prime}$ in Eq. (5)] and the spectral sidebands [caused by the $E_{1}^{\prime}$ and $E_{2}^{\prime}$ terms in Eq. (5)] in the excitation spectra of Rydberg $S_{1 / 2}$ and $P_{1 / 2}$ states of a single trapped ${ }^{88} \mathrm{Sr}^{+}$ion. We probe a Zeeman sublevel of a Rydberg $S_{1 / 2}$ state using two ultraviolet (UV) laser fields; a $243-\mathrm{nm}$ field couples the initial state $4 D_{5 / 2}$ with the intermediate state $6 P_{3 / 2}$, and a 306-nm field couples $6 P_{3 / 2}$ with $n S_{1 / 2}$. We probe a Zeeman sublevel of a Rydberg $P_{1 / 2}$ state using three fields: the two UV fields as well as a microwave field which couples Rydberg $S_{1 / 2}$ states to Rydberg $P_{1 / 2}$ states. Spectroscopy signals rely on the decay of states $6 P_{3 / 2}, n S_{1 / 2}$, and $n P_{1 / 2}$ to the ground state $5 S_{1 / 2}$; whether or not the ion is in the ground state is detected using ion fluorescence [20]. The entire process can be described as optical pumping from $4 D_{5 / 2} \rightarrow 5 S_{1 / 2}$. The level scheme is shown in Fig. 1 .

We use four different spectroscopy techniques. Three of the techniques are for probing $n S_{1 / 2}$ states, and the other one is for probing $n P_{1 / 2}$ states. Methods 2 and 3 offer resistance to ion loss by double ionization [27], while method 4 is well suited for probing weak resonances.

(1) Method 1 is for probing $n S_{1 / 2}$ : the 243-nm field is detuned from the $4 D_{5 / 2} \leftrightarrow 6 P_{3 / 2}$ resonance and the frequency of the 306-nm field is scanned. When a two-photon $4 D_{5 / 2} \leftrightarrow n S_{1 / 2}$ transition is resonant population is pumped from $4 D_{5 / 2} \rightarrow 5 S_{1 / 2}$ via $n S_{1 / 2}$.

(2) Method 2 is for probing $n S_{1 / 2}$ : a weak 243-nm field resonantly couples $4 D_{5 / 2} \leftrightarrow 6 P_{3 / 2}$ while the frequency of a strong 306-nm field is scanned. When the 306-nm field is not resonant to the $6 P_{3 / 2} \leftrightarrow n S_{1 / 2}$ transition population is pumped from $4 D_{5 / 2} \rightarrow 5 S_{1 / 2}$ via $6 P_{3 / 2}$. When the 306-nm field is near resonant it shifts the $6 P_{3 / 2}$ level, causing an Autler-Townes 
splitting which detunes the 243-nm field and reduces the rate of pumping $4 D_{5 / 2} \rightarrow 5 S_{1 / 2}$ via $6 P_{3 / 2}$.

(3) Method 3 is for probing $n^{\prime} P_{1 / 2}$ : building on method 2, the 243- and 306-nm fields are resonant while the frequency of the microwave field is scanned. When the microwave field is resonant to a $n S_{1 / 2} \leftrightarrow n^{\prime} P_{1 / 2}$ transition the $6 P_{3 / 2} \leftrightarrow n S_{1 / 2}$ coupling is weakened and the Autler-Townes splitting in the $4 D_{5 / 2} \leftrightarrow 6 P_{3 / 2}$ spectrum is broken, and the rate of pumping $4 D_{5 / 2} \rightarrow 5 S_{1 / 2}$ via $6 P_{3 / 2}$ is increased. Methods 2 and 3 rely on the opening and closing of the $4 D_{5 / 2} \rightarrow 5 S_{1 / 2}$ optical pumping path, depending on the frequency shifts due to dressing by the 306-nm laser field and the microwave field.

(4) Method 4 is for probing the $6 P_{3 / 2} \leftrightarrow n S_{1 / 2}$ coupling strength: a strong 306-nm field resonantly couples $6 P_{3 / 2} \leftrightarrow$ $n S_{1 / 2}$. The frequency of a weak 243-nm field is scanned; the resulting spectrum shows an Autler-Townes doublet the splitting of which reveals the $6 P_{3 / 2} \leftrightarrow n S_{1 / 2}$ coupling strength. This method is well suited for probing weak resonances.

Methods 1 and 4 were previously used in $[20,28]$, respectively. We introduce methods 2 and 3 , which offer resistance to losses through double ionization ${ }^{88} \mathrm{Sr}^{+} \rightarrow{ }^{88} \mathrm{Sr}^{2+}$ [27]. This advantage is described in detail in the Supplemental Material [11].

Although the initial $4 D_{5 / 2}$ state and intermediate $6 P_{3 / 2}$ state have permanent quadrupole moments we safely neglect the effects of the quadrupole fields on these states, since the effects on the $J=\frac{1}{2}$ Rydberg states are around six orders of magnitude larger.

Drifts of the strengths of the trapping quadrupole fields will cause quadrupole shifts $\left(E_{0}^{\prime}\right)$ and thus resonance frequencies to drift. We combat this by actively stabilizing the amplitude of the oscillating electric field in our system [29,30].

So that quadrupole effects dominate the Rydbergexcitation spectra, we mitigate Stark effects by minimizing the dipole electric field experienced by the ion. We achieve this by cooling the ion motion to the ground state $[18,20]$ and by minimizing ion micromotion [21].

\section{RESULTS}

\section{A. Static energy level shift}

The static energy level shift due to the quadrupole fields $\left[E_{0}^{\prime}\right.$ in Eq. (5)] means that Rydberg excitation frequencies depend on the field strengths. We investigate this by measuring the $4 D_{5 / 2} \leftrightarrow 56 S_{1 / 2}$ and $4 D_{5 / 2} \leftrightarrow 56 P_{1 / 2}$ resonance frequencies as the amplitude of the oscillating field $\phi_{\mathrm{rf}}$ is varied; the results are shown in Fig. 2. The energy required for excitation of $56 S_{1 / 2}$ depends quadratically on $\phi_{\mathrm{rf}}$ [Fig. 2(a)], according to the second-order perturbation description in Eq. (5); a quadratic fit returns $\alpha_{22}=(1.11 \pm$ $0.03) \times 10^{-44} \mathrm{~J} \mathrm{~m}^{4} \mathrm{~V}^{-2}$. The theory curve uses the theory value of the $56 S_{1 / 2}$ quadrupole polarizability $\alpha_{22}=1.18 \times$ $10^{-44} \mathrm{~J} \mathrm{~m}^{4} \mathrm{~V}^{-2}$. The experimental data in Figs. 2(a) and 2(b) were obtained using methods 1 and 3, respectively.

Rydberg $P_{1 / 2}$ states are generally more sensitive to quadrupole fields than Rydberg $S_{1 / 2}$ states, since the fields couple $P_{1 / 2}$ states to the energetically nearby $P_{3 / 2}$ states (as well as more distant $F_{5 / 2}$ states), while the fields only couple $S_{1 / 2}$ states to the more distant $D$ states. The higher sensitivity

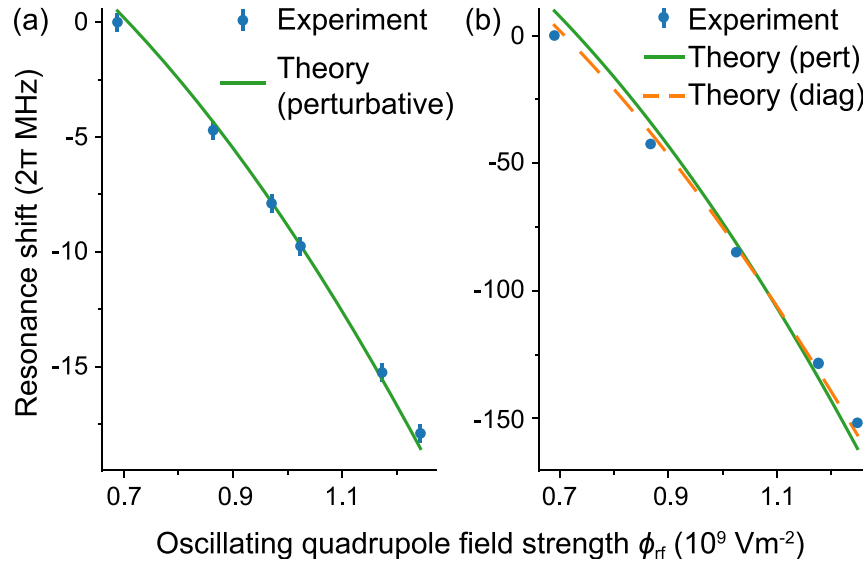

FIG. 2. Rydberg state energies depend on the strength of the oscillating quadrupole field $\phi_{\mathrm{rf}}$. (a) The response of $56 S_{1 / 2}$ to $\phi_{\mathrm{rf}}$ is described by the second-order perturbative calculation. (b) The response of $56 P_{1 / 2}$ to $\phi_{\mathrm{rf}}$ is described better by the full diagonalization calculation than by the second-order perturbative calculation. Error bars representing uncertainties in resonance frequencies at the $68 \%$ confidence level are visible in (a) while they are smaller than the markers in (b).

of $56 P_{1 / 2}$ relative to $56 S_{1 / 2}$ is seen by comparing Fig. 2(b) with Fig. 2(a). The solid curve shows the response calculated using second-order perturbation theory, which returns $\alpha_{22}=1.05 \times 10^{-43} \mathrm{~J} \mathrm{~m}^{4} \mathrm{~V}^{-2}$ for $56 P_{1 / 2}$. The strong response of $56 P_{1 / 2}$ to $\phi_{\mathrm{rf}}$ means that terms in the perturbation expansion that are higher than second order cannot be neglected. Instead of using higher-order perturbation calculations, we capture the higher-order terms by full diagonalization of the Hamiltonian (details in the Supplemental Material [11]). The full diagonalization calculation (dashed curve) describes the experimental data better than the second-order perturbation calculation. The full diagonalization calculation results do not describe the experimental data perfectly; this could be because Stark effects are not considered, and Stark effects were not completely mitigated in the experiment.

\section{B. Oscillating energy level shift}

The oscillating quadrupole field $\phi_{\mathrm{rf}}$ causes an oscillating quadrupole shift [Eq. (5)], which leads to sidebands in Rydberg-excitation spectra, as shown in Figs. 3(a) and 3(b). Two sets of sidebands can appear: the modulation with frequency $\Omega$ causes sidebands at multiples of $\Omega$ described by modulation index $\beta_{1}=\frac{E_{1}^{\prime}}{\hbar \Omega}$ while the modulation with frequency $2 \Omega$ causes sidebands at multiples of $2 \Omega$ described by modulation index $\beta_{2}=\frac{E_{2}^{\prime}}{2 \hbar \Omega}$. In a linear Paul trap the oscillating field strength is usually much larger than the strengths of the static fields $\phi_{\mathrm{rf}} \gg \phi_{\mathrm{ax}}, \phi_{\mathrm{rad}}$ and $E_{0}^{\prime} \approx E_{2}^{\prime} \gg E_{1}^{\prime}$ and $\beta_{2} \gg \beta_{1}$. This means the sidebands at multiples of $2 \Omega$ are much larger than the sidebands at multiples of $\Omega$. We operate our trap in this regime. The experimental data in Figs. 3(a) and 3 (b) were obtained using methods 2 and 3, respectively.

The $4 D_{5 / 2} \leftrightarrow 56 S_{1 / 2}$ spectrum in Fig. 3(a) displays a saturated carrier peak and weak sidebands due to the $2 \Omega$ modulation. No sidebands due to the much weaker $\Omega$ modulation are visible. Good agreement is observed between the 

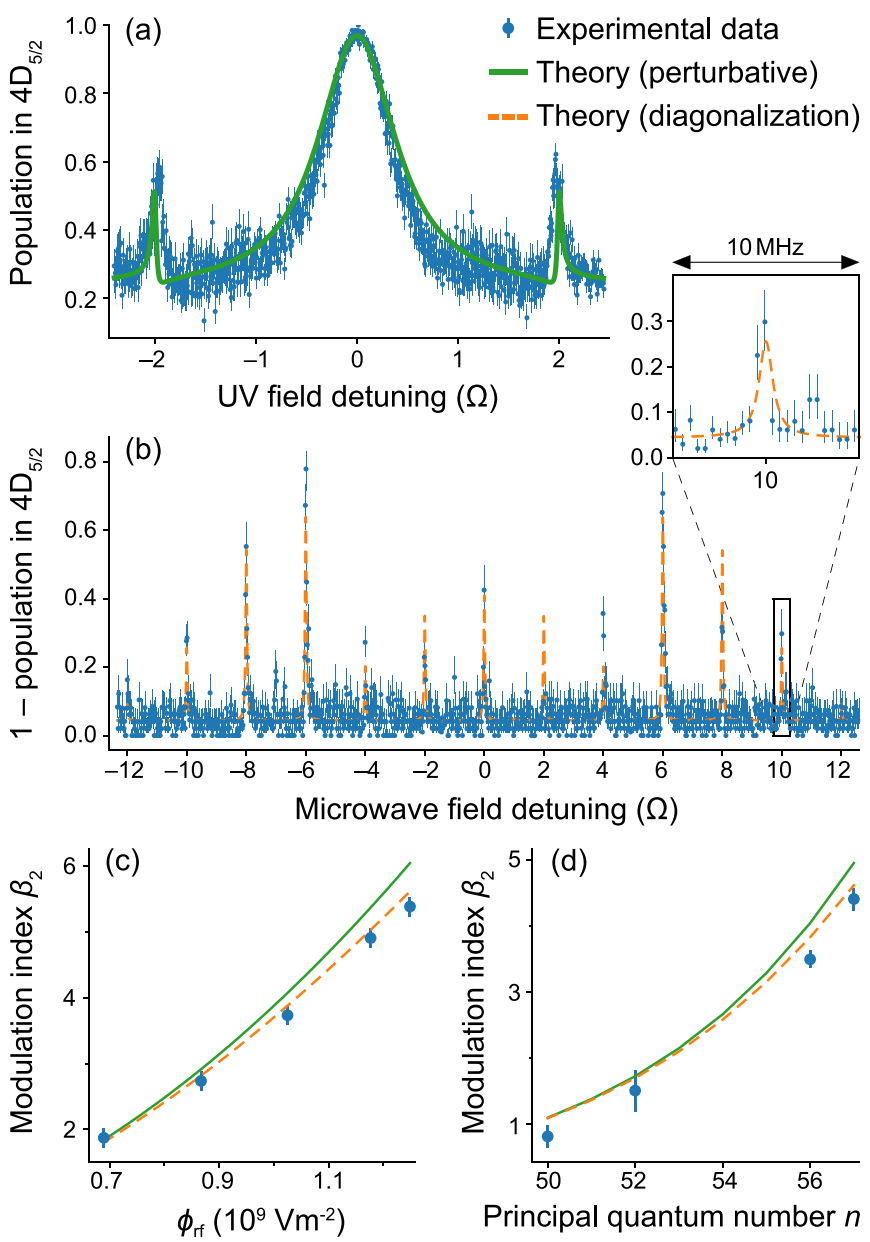

FIG. 3. The oscillating quadrupole shift introduces sidebands to Rydberg-excitation spectra. (a) The $56 S_{1 / 2}$ excitation spectrum displays weak $2 \Omega$ sidebands. (b) The $57 P_{1 / 2}$ excitation spectrum displays a forest of sidebands. The inset shows a $10-\mathrm{MHz}$ section of the spectrum around $10 \Omega$ detuning. (c) The modulation index $\beta_{2}$ (which describes the sideband amplitudes) increases with the amplitude of the oscillating quadrupole field $\phi_{\mathrm{rf}}$; state $56 P_{1 / 2}$ was used. (d) $\beta_{2}$ increases with the principal quantum number $n$ of Rydberg $P_{1 / 2}$ states. In (c) and (d) the measured $\beta_{2}$ is described better by the full diagonalization calculation than by the second-order perturbative calculation. Throughout this Rapid Communication $\Omega=2 \pi \times 18.1 \mathrm{MHz}$. Error bars represent $68 \%$ confidence intervals; in (a) and (b) error bars represent quantum projection noise; in (c) and (d) error bars represent uncertainties in extracting $\beta_{2}$ from spectra.

experimental data and the theory curve: the theory curve uses the calculated $56 S_{1 / 2}$ quadrupole polarizability and experimentally determined parameters (the sole free parameter is the resonance frequency).

Before we discuss $n P_{1 / 2}$ spectra we refine a point made earlier: Due to the technique we use to probe $n P_{1 / 2}$ states (method 3), the $n P_{1 / 2}$ excitation spectra are described by a modulation index which depends on the difference between the $n S_{1 / 2}$ and $n P_{1 / 2}$ modulations $\beta_{2}=\frac{E_{2, P}^{\prime}-E_{2, S}^{\prime}}{2 \hbar \Omega}$. This is corroborated by simulations in the Supplemental Material [11].

Note that $57 P_{1 / 2}$ is much more sensitive to quadrupole fields than $56 S_{1 / 2}$; using the same trap settings as in Fig. 3(a) the $4 D_{5 / 2} \leftrightarrow 57 P_{1 / 2}$ spectrum appears as a forest of resonance peaks, shown in Fig. 3(b). The relative strengths of the resonances are well described by Bessel functions of the first kind $\left[J_{m}\left(\beta_{2}\right)\right]^{2}$ at the $m$ th multiple of $2 \Omega$, with $\beta_{2}=4.62$. This value of $\beta_{2}$ was found by full diagonalization of the Hamiltonian (details in the Supplemental Material [11]). The experimental data are described well by the theory curve, in which the relative strengths of the resonances are $\left[J_{m}\left(\beta_{2}\right)\right]^{2}$ and the positions are $2 m \Omega$.

The modulation with frequency $\Omega$ is too weak to introduce significant sidebands in Fig. 3(a) or Fig. 3(b). If the $\Omega$ modulation was stronger, its interplay with the $2 \Omega$ modulation would introduce a spectral asymmetry.

Note that $56 P_{1 / 2}$ excitation spectra were measured as the amplitude of the oscillating quadrupole field $\phi_{\mathrm{rf}}$ was varied. The modulation index $\beta_{2}$ was extracted from the spectra and its dependence on $\phi_{\mathrm{rf}}$ is shown in Fig. 3(c). The full diagonalization calculation describes the experimental data better than the second-order perturbation calculation, as was the case for the data in Fig. 2(b).

Excitation spectra of Rydberg $P_{1 / 2}$ states with principal quantum numbers $n$ between 50 and 57 were measured; the quadrupole field strengths were kept fixed. $\beta_{2}$ values were extracted and the dependence of $\beta_{2}$ on $n$ is shown in Fig. 3(d). The response of Rydberg $P_{1 / 2}$ states to quadrupole fields is captured better by the full diagonalization calculation than by the second-order perturbative approach, just as for the data in Figs. 2(b) and 3(c). The perturbation theory curve scales with the effective principal quantum number $n^{*}$ as $n^{* 11}$; this is the expected scaling of the quadrupole polarizability (this comes from second-order perturbation theory: quadrupole couplings grow with $n^{* 4}$, the square of the couplings contributes to the second-order perturbation, and energy splittings decay as $n^{*-3}$ ).

The oscillating energy shift [Eq. (5)] is challenging to quantitatively investigate when the modulation index $\beta$ is small, as is the case for Rydberg $S_{1 / 2}$ states. Using spectroscopy the first sideband is probed more weakly than the carrier by the factor $\left[\frac{J_{1}(\beta)}{J_{0}(\beta)}\right]^{2} \approx \frac{\beta^{2}}{4}$; this is the case in Fig. 3(a) where $\beta_{2}=0.3$. Coherent spectroscopy techniques (such as method 4) can offer a more favorable approach when $\beta$ is small; with some coherent spectroscopy the effect of the first sideband transition is smaller than the effect of the carrier by $\frac{J_{1}(\beta)}{J_{0}(\beta)} \approx \frac{\beta}{2}$.

We use the Autler-Townes effect (method 4) to probe weak modulations, as shown in Fig. 4.

When the Rydberg $S_{1 / 2}$ energy level is not modulated ( $\beta=$ 0 ) the 306-nm laser field resonantly couples $6 P_{3 / 2} \leftrightarrow n S_{1 / 2}$ with strength $\Omega_{c}$. When there is a modulation the coupling strength is $J_{0}(\beta) \Omega_{c}$, and the $4 D_{5 / 2} \leftrightarrow 6 P_{3 / 2}$ excitation spectrum displays two resonance peaks split by $J_{0}(\beta) \Omega_{c}$ [see Fig. 4(a)]. If the 306-nm laser field is detuned such that it couples $6 P_{3 / 2}$ and $n S_{1 / 2}$ via the first sideband transition, this coupling then has strength $J_{1}(\beta) \Omega_{c}$, and the peaks in the $4 D_{5 / 2} \leftrightarrow 6 P_{3 / 2}$ spectrum are split by $J_{1}(\beta) \Omega_{c}$ [see Fig. 4(b)]. The ratio of the two splittings is $\frac{J_{1}(\beta)}{J_{0}(\beta)} \approx \frac{\beta}{2}$ (the approximation is valid for $\beta \ll 1$ ). From such measurements we determine the $46 S_{1 / 2}$ quadrupole polarizability $\alpha_{22}=(1.08 \pm 0.05) \times$ $10^{-45} \mathrm{~J} \mathrm{~m}^{4} \mathrm{~V}^{-2}$, which is similar to the calculated value $\alpha_{22}=$ $1.21 \times 10^{-45} \mathrm{~J} \mathrm{~m}^{4} \mathrm{~V}^{-2}$. 


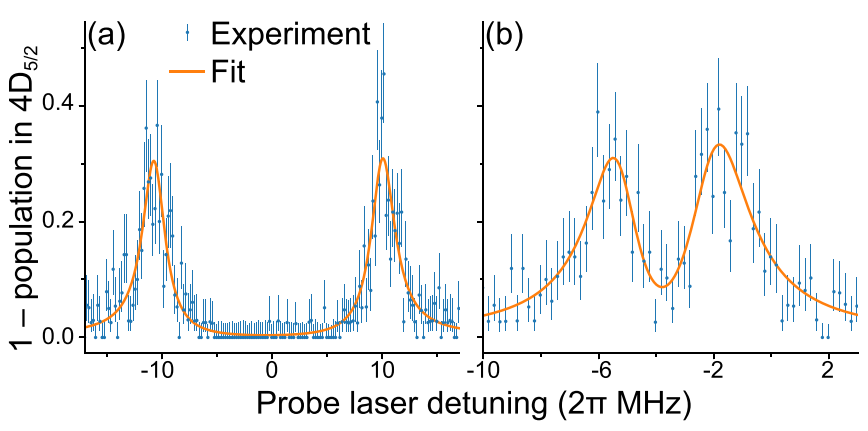

FIG. 4. The oscillating quadrupole shift is probed using the Autler-Townes effect. The oscillating quadrupole shift introduces sidebands $\pm 2 \Omega$ from the $6 P_{3 / 2} \leftrightarrow 46 S_{1 / 2}$ resonance. The sideband coupling strength is compared with the carrier coupling strength by comparing the Autler-Townes splittings that they each induce on the weakly probed $4 D_{5 / 2} \leftrightarrow 6 P_{3 / 2}$ transition. In (a) the coupling laser is resonant to the $6 P_{3 / 2} \leftrightarrow 46 S_{1 / 2}$ carrier transition; in (b) the coupling laser is resonant to a sideband transition. Error bars represent quantum projection noise ( $68 \%$ confidence intervals).

\section{v. CONCLUSIONS}

Systems of trapped Rydberg ions have recently offered a new approach to quantum computation and simulation [16]. However, unwanted coupling to quadrupole-field-induced sidebands may reduce the fidelity of a Rydberg ion quantum gate [31]. Proposals for reducing or removing this coupling include tuning $\phi_{\mathrm{rf}}$ such that $J_{ \pm 1}\left(\beta_{2}\right)=0$ and the first-order sidebands at $\pm 2 \Omega$ vanish, using a higher value of $\Omega$ to diminish $\beta_{2}$ [32], and modulating the Rydberg-excitation laser fields and microwave field such that these fields follow the oscillating Rydberg energy levels; alternatively one could confine ions using a rotating Paul trap [33,34], a Penning trap [35], or a digital ion trap [36] operating with periods of zero field strength and excite Rydberg ions during these periods [14].

In this Rapid Communication we investigate higher-order effects of electric quadrupole fields on a single ion. Effects on Rydberg $S_{1 / 2}$ states are described well in terms of the electric quadrupole polarizability. Rydberg $P_{1 / 2}$ states are more sensitive to quadrupole fields, and full diagonalization calculations describe the response of $P_{1 / 2}$ states better than the second-order perturbation calculations. The resonance shifts and spectral sidebands we observe will need to be considered in future experiments involving highly sensitive Rydberg atoms, molecules, or ions in ion traps. Additionally the resonance shifts may become important in future trapped ion precision experiments and clocks $[9,10]$, as shown in the Supplemental Material [11].

The huge dipole polarizabilities of trapped Rydberg ions offer a range of applications [21,37-41]. We hope that this Rapid Communication will stimulate new research avenues which take advantage of the extreme quadrupole polarizabilities of trapped Rydberg ions; for instance, the sensitivity of Rydberg ions to oscillating quadrupole fields may allow for Floquet engineering [42].

\section{ACKNOWLEDGMENTS}

We thank Cornelius Hempel for information about trap stabilization, Shinsuke Haze and Markus Deiß for information about hybrid atom-ion systems and digital ion traps, Milena Guevara-Bertsch for information about trapped ion precision spectroscopy, and all members of the Entangled Rydberg matter for quantum sensing and simulations (ERyQSenS) consortium for discussions. This work was supported by the European Research Council under the European Union's Seventh Framework Programme Grant No. 279508, the Swedish Research Council (Trapped Rydberg Ion Quantum Simulator), the QuantERA ERA-NET Cofund in Quantum Technologies (ERyQSenS), and the Knut and Alice Wallenberg Foundation (Photonic Quantum Information and through the Wallenberg Centre for Quantum Technology [WACQT]).

C.Z. first measured and interpreted the resonance shift; G.H. and C.Z. carried out the calculations; C.Z. developed the spectroscopy technique for Rydberg $P$ states; G.H., F.P., C.Z., H.P., E.J., and S.S. carried out the measurements; G.H. wrote the manuscript; M.H. supervised the project; all authors contributed to the discussion of the results and to the manuscript.

G.H. and C.Z. contributed equally to this work.
[1] S. Weber, C. Tresp, H. Menke, A. Urvoy, O. Firstenberg, H. P. Büchler, and S. Hofferberth, Calculation of Rydberg interaction potentials, J. Phys. B 50, 133001 (2017).

[2] X. Han, S. Bai, Y. Jiao, L. Hao, Y. Xue, J. Zhao, S. Jia, and G. Raithel, Cs $62 D_{J}$ Rydberg-atom macrodimers formed by long-range multipole interaction, Phys. Rev. A 97, 031403(R) (2018).

[3] C. G. Gray and K. E. Gubbins, Theory of Molecular Fluids (Clarendon, Oxford, 1984), Vol. 1.

[4] B. Verberck, K. H. Michel, and A. V. Nikolaev, Crystal structures of polymerized fullerides $\mathrm{AC}_{60}, \mathrm{~A}=\mathrm{K}, \mathrm{Rb}, \mathrm{Cs}$, and alkali-mediated interactions, J. Chem. Phys 116, 10462 (2002).

[5] G. Benedek and P. Toennies, Atomic Scale Dynamics at Surfaces (Springer-Verlag, Berlin, 2018).

[6] I. M. Dimitrova, R. I. Slavchov, T. Ivanov, and S. Mosbach, A spherical cavity model for quadrupolar dielectrics, J. Chem. Phys 144, 114502 (2016).
[7] F. Bernal Arango, T. Coenen, and A. F. Koenderink, Underpinning hybridization intuition for complex nanoantennas by magnetoelectric quadrupolar polarizability retrieval, ACS Photonics 1, 444 (2014).

[8] W. M. Itano, External-field shifts of the ${ }^{199} \mathrm{Hg}^{+}$optical frequency standard, J. Res. Nat. Inst. Stand. Technol. 105, 829 (2000).

[9] A. D. Ludlow, M. M. Boyd, J. Ye, E. Peik, and P. O. Schmidt, Optical atomic clocks, Rev. Mod. Phys. 87, 637 (2015).

[10] K. J. Arnold, R. Kaewuan, T. R. Tan, and M. D. Barrett, Oscillating quadrupole effects in high-precision metrology, Phys. Rev. A 99, 022515 (2019).

[11] See Supplemental Material at http://link.aps.org/supplemental/ 10.1103/PhysRevResearch.3.L032032 for information about the influence of second-order quadrupole effects on trapped ion precision spectroscopy, perturbative calculation of the quadrupole polarizability, spectroscopy methods 2 
and 3, full diagonalization calculations, and quadrupole sidebands in Rydberg-P state excitation spectra, which includes Refs. [43,44].

[12] S. G. Porsev, M. S. Safronova, U. I. Safronova, and M. G. Kozlov, Multipolar Polarizabilities And Hyperpolarizabilities In The Sr Optical Lattice Clock, Phys. Rev. Lett. 120, 063204 (2018).

[13] N. V. Ewald, T. Feldker, H. Hirzler, H. A. Fürst, and R. Gerritsma, Observation Of Interactions Between Trapped Ions And Ultracold Rydberg Atoms, Phys. Rev. Lett. 122, 253401 (2019).

[14] S. Haze, J. Wolf, M. Deiß, L. Wang, G. Raithel, and J. Hecker Denschlag, Stark spectroscopy of Rydberg atoms in an atomion hybrid trap, arXiv:1901.11069 (2019).

[15] M. Deiß, S. Haze, J. Wolf, L. Wang, F. Meinert, C. Fey, F. Hummel, P. Schmelcher, and J. Hecker Denschlag, Observation of spin-orbit-dependent electron scattering using long-range Rydberg molecules, Phys. Rev. Research 2, 013047 (2020).

[16] C. Zhang, F. Pokorny, W. Li, G. Higgins, A. Pöschl, I. Lesanovsky, and M. Hennrich, Submicrosecond entangling gate between trapped ions via Rydberg interaction, Nature (London) 580, 345 (2020).

[17] M. P. A. Jones, R. M. Potvliege, and M. Spannowsky, Probing new physics using Rydberg states of atomic hydrogen, Phys. Rev. Res. 2, 013244 (2020).

[18] M. Müller, L. Liang, I. Lesanovsky, and P. Zoller, Trapped Rydberg ions: from spin chains to fast quantum gates, New J. Phys. 10, 093009 (2008).

[19] T. Feldker, P. Bachor, M. Stappel, D. Kolbe, R. Gerritsma, J. Walz, and F. Schmidt-Kaler, Rydberg Excitation Of A Single Trapped Ion, Phys. Rev. Lett. 115, 173001 (2015).

[20] G. Higgins, W. Li, F. Pokorny, C. Zhang, F. Kress, C. Maier, J. Haag, Q. Bodart, I. Lesanovsky, and M. Hennrich, Single Strontium Rydberg Ion Confined In A Paul Trap, Phys. Rev. X 7, 021038 (2017)

[21] G. Higgins, F. Pokorny, C. Zhang, and M. Hennrich, Highly Polarizable Rydberg Ion in a Paul Trap, Phys. Rev. Lett. 123, 153602 (2019).

[22] M. Pawlak and H. R. Sadeghpour, Rydberg spectrum of a single trapped $\mathrm{Ca}^{+}$ion: A Floquet analysis, Phys. Rev. A 101, 052510 (2020).

[23] W. Li and I. Lesanovsky, Entangling quantum gate in trapped ions via Rydberg blockade, Appl. Phys. B 114, 37 (2014).

[24] Fabian Pokorny, Chi Zhang, Gerard Higgins, and Markus Hennrich, Magic trapping of a Rydberg ion with a diminished static polarizability, arXiv:2005.12422 (2020).

[25] C. G. Gray and B. W. N. Lo, Spherical tensor theory of molecular multipole moments and polarizabilities, Chem. Phys. 14, 73 (1976).

[26] D. Leibfried, R. Blatt, C. Monroe, and D. Wineland, Quantum dynamics of single trapped ions, Rev. Mod. Phys. 75, 281 (2003).

[27] G. Higgins, A Single Trapped Rydberg Ion (Springer, New York, 2019).
[28] G. Higgins, F. Pokorny, C. Zhang, Q. Bodart, and M. Hennrich, Coherent Control of a Single Trapped Rydberg Ion, Phys. Rev. Lett. 119, 220501 (2017).

[29] Cornelius Hempel, Digital quantum simulation, Schrödinger cat state spectroscopy and setting up a linear ion trap, $\mathrm{Ph} . \mathrm{D}$. thesis, Universität Innsbruck, 2014.

[30] K. G. Johnson, J. D. Wong-Campos, A. Restelli, K. A. Landsman, B. Neyenhuis, J. Mizrahi, and C. Monroe, Active stabilization of ion trap radiofrequency potentials, Rev. Sci. Instrum 87, 053110 (2016).

[31] F. Schmidt-Kaler, T. Feldker, D. Kolbe, J. Walz, M. Müller, P. Zoller, W. Li, and I. Lesanovsky, Rydberg excitation of trapped cold ions: A detailed case study, New J. Phys. 13, 075014 (2011).

[32] L. S. Simeonov, N. V. Vitanov, and P. A. Ivanov, Compensation of the trap-induced quadrupole interaction in trapped Rydberg ions, Sci. Rep 9, 7340 (2019).

[33] T. Hasegawa and J. J. Bollinger, Rotating-radio-frequency ion traps, Phys. Rev. A 72, 043403 (2005).

[34] Chi Zhang, Fast and scalable entangling gate in trapped ions via Rydberg interaction, Ph.D. thesis, Stockholm University, 2020.

[35] L. S. Brown and G. Gabrielse, Geonium theory: Physics of a single electron or ion in a Penning trap, Rev. Mod. Phys. 58, 233 (1986).

[36] N. Deb, L. L. Pollum, A. D. Smith, M. Keller, C. J. Rennick, B. R. Heazlewood, and T. P. Softley, Coulomb crystal mass spectrometry in a digital ion trap, Phys. Rev. A 91, 033408 (2015).

[37] W. Li and I. Lesanovsky, Electronically Excited Cold Ion Crystals, Phys. Rev. Lett. 108, 023003 (2012).

[38] W. Li, A. W. Glaetzle, R. Nath, and I. Lesanovsky, Parallel execution of quantum gates in a long linear ion chain via Rydberg mode shaping, Phys. Rev. A 87, 052304 (2013).

[39] R. Nath, M. Dalmonte, A. W. Glaetzle, P. Zoller, F. SchmidtKaler, and R. Gerritsma, Hexagonal plaquette spin-spin interactions and quantum magnetism in a two-dimensional ion crystal, New J. Phys. 17, 065018 (2015).

[40] J. Vogel, W. Li, A. Mokhberi, I. Lesanovsky, and F. SchmidtKaler, Shuttling Of Rydberg Ions For Fast Entangling Operations, Phys. Rev. Lett. 123, 153603 (2019).

[41] F. M. Gambetta, I. Lesanovsky, and W. Li, Exploring nonequilibrium phases of the generalized Dicke model with a trapped Rydberg-ion quantum simulator, Phys. Rev. A 100, 022513 (2019).

[42] T. Oka and S. Kitamura, Floquet engineering of quantum materials, Annu. Rev. Condens. Matter Phys 10, 387 (2019).

[43] N. Šibalić, J. D. Pritchard, C. S. Adams, and K. J. Weatherill, ARC: An open-source library for calculating properties of alkali Rydberg atoms, Comput. Phys. Commun. 220, 319 (2017).

[44] J. Andrijauskas, J. Vogel, A. Mokhberi, and F. SchmidtKaler, Precision measurement of the ionization energy of a single trapped ${ }^{40} \mathrm{Ca}^{+}$ion by Rydberg series excitation, arXiv:2009.01070 (2020). 